\title{
CÁNCER DE PRÓSTATA METASTÁSICO ASOCIADO A VALORES BAJOS DE ANTÍGENO PROSTÁTICO ESPECÍFICO
}

\author{
Silvia Diaz ${ }^{1, a}$, Michael Salirrosas ${ }^{1, b}$
}

\begin{abstract}
RESUMEN
Se reporta el caso de un paciente de 67 años que presenta dolor en el glúteo derecho que se irradia hacia los muslos afectando la bipedestación y la marcha. Con tiempo de enfermedad de cuatro meses, asociado con la disminución de $15 \mathrm{~kg}$ de peso; se realizaron exámenes de imágenes donde se encontró un proceso infiltrativo de hueso sacro. La biopsia se informó como lesión de tejido óseo infiltrado por adenocarcinoma poco diferenciado. La evaluación urológica clínica, a pesar del valor del antígeno prostático específico (PSA total: 4,62 mg/dL), encontró una lesión nodular menor a un centímetro en el examen de tacto rectal. La biopsia de próstata evidenció un adenocarcinoma pobremente diferenciado (score de Gleason: 8). La gammagrafía ósea mostró lesiones activas relacionadas con metástasis en tercio medio de clavícula derecha, hueso sacro y región púbica derecha.
\end{abstract}

Palabras clave: Cáncer de próstata; Antígeno prostático específico; Metástasis de la neoplasia (fuente: DeCS BIREME).

\section{METASTATIC PROSTATE CANCER ASSOCIATED WITH LOW LEVELS OF PROSTATE-SPECIFIC ANTIGEN}

\begin{abstract}
A case was reported of a 67 -year-old patient with right buttock pain radiating to the thighs and affecting his bipedalism and gait. With four months into the disease and a weight loss of 15 kilos, the patient underwent imaging tests which showed sacrum infiltration. The biopsy diagnosis was injury of bone tissue infiltrated by poorly differentiated adenocarcinoma. The rectal examination performed as part of the clinical urologic examination revealed a nodular lesion of less than one centimeter, despite the level of prostate-specific antigen (total PSA: $4.62 \mathrm{mg} / \mathrm{dL}$ ). The prostate biopsy evidenced a poorly differentiated adenocarcinoma (Gleason score: 8 ). The bone scan showed active lesions associated with metastasis in the middle third of the right clavicle, the sacrum and the right pubic region.
\end{abstract}

Key words: Prostatic neoplasms; Prostate-specific antigen; Neoplasm metastasis (source: MeSH NLM).

\section{INTRODUCCIÓN}

El cáncer de próstata representa el tumor sólido maligno más frecuente en el varón y es el segundo como causa de muerte en los hombres ${ }^{(1)}$. Las lesiones por carcinoma de próstata pueden ser reconocidas durante el examen de tacto rectal dada su ubicación preferente en la zona periférica de la próstata. Sin embargo, solo el $18 \%$ de todos los pacientes con cáncer de próstata son detectados por este examen (2).
El antígeno prostático específico (PSA) es una proteína producida exclusivamente por las células epiteliales de la próstata. Descubierta y utilizada desde fines de la década de los ochenta, ha servido para detectar un mayor número de casos de cáncer de próstata, especialmente en pacientes asintomáticos que corresponden a estadios más tempranos de enfermedad (3-5). En la mayoría de casos el valor de PSA se eleva según progresa la enfermedad, siendo infrecuente los casos reportados con metástasis y valores bajos de PSA ${ }^{(6)}$. Se presenta

\footnotetext{
Centro Médico Naval. Callao, Perú

a Médico internista, magister en Medicina con mención en Medicina Interna; ${ }^{\mathrm{b}}$ médico urólogo

Recibido: 12-03-12 Aprobado: 31-10-12
}

Citar como: Diaz S, Salirrosas M. Cáncer de próstata metastásico asociado a valores bajos de antígeno prostático específico. Rev Peru Med Exp Salud Publica. 2012;29(4):571-4. 
el caso clínico de un paciente con cáncer de próstata metastásico con valores bajos de PSA.

\section{REPORTE DE CASO}

Paciente varón, 67 años, natural de Lima, procedente del Callao, militar retirado, hospitalizado en el Centro Médico Naval en noviembre de 2009, con un tiempo de enfermedad de cuatro meses, caracterizada por dolor intenso tipo punzante en región glútea derecha que irradia a cara posterior de muslo derecho. El dolor aumentaba con la marcha y la sedestación prolongada.

Tres meses antes del ingreso, por prescripción médica para el dolor (en región lumbar), recibió sesiones de terapia física durante un mes sin obtener mejoría, y continuó con medicación oral y parenteral con diclofenaco y orfenadrina. Dos meses antes del ingreso presentó dolor en el sacro irradiado a ambos miembros inferiores que, progresivamente, le impidieron la deambulación. Asimismo, se evidenció una disminución de peso en $15 \mathrm{~kg}$, a partir del inicio de la enfermedad.

Presenta el antecedente que en consulta ambulatoria de urología en julio de 2009, se difirió el examen de tacto rectal por presentar intenso dolor durante la evaluación y referir sensación de evacuación incompleta y heces acompañadas de sangre. Se le derivó al servicio de gastroenterología indicándole una proctoscopia rígida (octubre 2009), en la que se encontró una fisura anal crónica y hemorroides externas pequeñas.
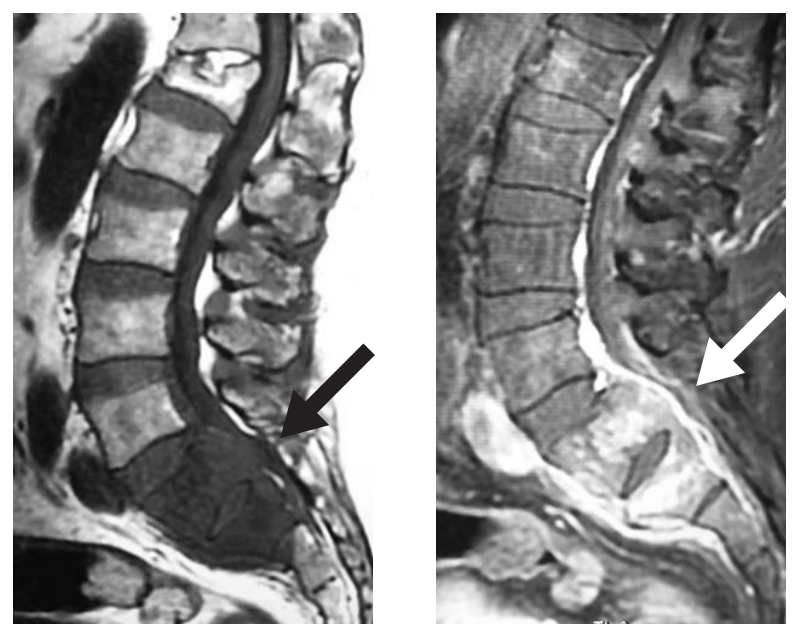

Figura 1. (A) Resonancia magnética de columna lumbosacra muestra proceso infiltrativo que compromete el hueso sacro (S1 y S2) con hipointensidad en T1 (flecha negra). (B) El proceso infiltrativo en hueso sacro (S1 y S2) alcanza brillantez heterogénea luego de la inyección de sustancia de contraste (flecha blanca).
Otros antecedentes del paciente: hiperplasia prostática benigna diagnosticada dos años antes y en tratamiento con tamsulosina $(0,4 \mathrm{mg} / \mathrm{día})$, y extracto de Sabal serrulata (1 tableta bid); PSA:4 ng/mL (diciembre 2008); tuberculosis pulmonar hace 43 años; fractura vertebral de primera lumbar por accidente de tránsito hace 25 años; hipertensión arterial diagnosticada hace 5 años, y hernioplastía inguinal hace 5 años.

Al examen físico se encontró regular estado general, posición antálgica en decúbito lateral. Presión arterial, 120/70 mmHg; frecuencia cardiaca, 74 latidos por minuto; frecuencia respiratoria, 18 respiraciones por minuto; temperatura oral, $37^{\circ} \mathrm{C}$. Evaluación pulmonar, cardiovascular y de abdomen sin alteraciones. Puntos renoureterales y puñopercusión lumbar negativa. Tacto rectal diferido por dolor. Evaluación neurológica: lúcido, con lenguaje coherente y finalístico. Gran limitación axial a predominio de flexión por dolor. Dandy positivo a nivel sacro. Reflejo aquiliano bilateral abolido. Dolor a la presión digital en cuadrado lumbar, glúteo mayor y gemelo derecho.

Los exámenes auxiliares determinaron: hemograma, 7000 leucocitos (segmentados, $74 \%$; linfocitos, $20 \%$; monocitos, $5 \%$; eosinófilos, $1 \%$ ); hemoglobina, $12,8 \mathrm{~g} \%$; hematocrito, 33\%; plaquetas: 258000 por $\mathrm{mm}^{3}$; glucosa, $91 \mathrm{mg} / \mathrm{dL}$; creatinina, $1,0 \mathrm{mg} / \mathrm{dL}$; urea, $26 \mathrm{mg} / \mathrm{dL}$; examen completo de orina sin alteraciones; VSG, $48 \mathrm{~mm} / \mathrm{h}$; proteína C reactiva, $3,76 \mathrm{mg} / \mathrm{mL}(\mathrm{VN}: 0-3 \mathrm{mg} / \mathrm{mL})$; fosfatasa alcalina, $137 \mathrm{U} / \mathrm{L}(\mathrm{VN}: 40-180 \mathrm{U} / \mathrm{L})$; transaminasas normales; albúmina, $3,8 \mathrm{~g} / \mathrm{mL}$; globulinas, $2,7 \mathrm{~g} / \mathrm{mL}$; calcio sérico, $8,9 \mathrm{mg} / \mathrm{mL}$. La radiografía lumbosacra mostró aplastamiento del cuerpo vertebral L1 y pinzamiento de vértebras L5-S1.

Durante la hospitalización el paciente cursa con dolor intenso que no se controla con antiinflamatorios no esteroideos, se logró mejoría al iniciar opiodes (tramadol). Al ampliar estudios por el síndrome consuntivo, la tomografía abdominopélvica revela un proceso neoformativo de hueso sacro que parecía comprometer partes blandas. La resonancia magnética confirma proceso infiltrativo que compromete a los cuerpos vertebrales sacros $\mathrm{S} 1$ y $\mathrm{S} 2$ con hipointensidad en T1 y T2 (Figura 1A) y alcanzando brillantez heterogénea luego de la inyección de sustancia de contraste (Figura 1B). Dicho proceso se expande hacia la pelvis $y$, posteriormente, ocluye el canal raquídeo y se expande hacia ambos lados de la línea media comprometiendo los alerones sacros, siendo el lado izquierdo el de mayor compromiso.

Los resultados de marcadores tumorales mostraron: PSA total, 4,62 ng/mL; PSA libre, 0,16 ng/ mL, antígeno 
carcinoembrionario, $3,33 \mathrm{ng} / \mathrm{mL}$ ( $\mathrm{VN}$ : 0-5), alfafetoproteína, $0,96 \mathrm{ng} / \mathrm{mL}(\mathrm{VN}: 0-10)$. Se le realizó una biopsia de la lesión sacra. El informe de anatomía patológica concluyó tejido óseo infiltrado por neoplasia maligna indiferenciada. Al examen inmunohistoquímico, queratina de bajo peso molecular positivo correspondiente a adenocarcinoma poco diferenciado.

Es evaluado en el servicio de urología y se logra realizar el tacto rectal donde se encontró un nódulo menor a un $1 \mathrm{~cm}$ de diámetro en el lóbulo derecho; se realizó una biopsia de próstata. El informe de anatomía patológica reveló adenocarcinoma pobremente diferenciado, Gleason $4+4=8 / 10$. La neoplasia compromete el $50 \%$ de la muestra, permeación perineural presente. La gammagrafía ósea mostró lesiones activas relacionadas con metástasis en tercio medio de clavícula derecha, hueso sacro y región púbica derecha.

El paciente es derivado al servicio de oncología iniciándole tratamiento con bicalutamida $150 \mathrm{mg} / \mathrm{día}$ y acetato de leuprolide $7,5 \mathrm{mg} / \mathrm{mes}$ y bifosfonatos. A los dos meses presentó mejoría del dolor, recuperación de peso y disminución de los niveles de PSA total a $0,04 \mathrm{ng} / \mathrm{mL}$ a los 5 meses. Sin embargo, evolucionó a los dos años con invasión del cono medular por lo que recibió radioterapia.

\section{DISCUSIÓN}

El nivel de PSA es una variable independiente como mejor predictor de cáncer de próstata en comparación con el tacto rectal y la ecografía transrectal ${ }^{(7)}$. El valor de PSA mayor de $4 \mathrm{ng} / \mathrm{mL}$ se considera como punto de corte de detección de cáncer de próstata en vista del incremento evidente de la detección del cáncer a partir de este valor ${ }^{(3)}$. Sin embargo, en un estudio sobre prevención de cáncer de próstata en Estados Unidos se siguió a pacientes mayores de 55 años con PSA entre 0,5 a $4 \mathrm{ng} / \mathrm{mL}$ con tacto rectal normal por un lapso de 7 años, se les realizó biopsia al final del estudio (incluso al grupo control), y se encontró cáncer en el 6,6 a $26,9 \%$. Es decir, podemos encontrar cáncer en estadio temprano con valores bajos de PSA ${ }^{(8)}$. Se ha postulado que la transcripción del gen del PSA es regulado positivamente por el receptor androgénico, por lo que una deleción de este receptor podría ser el responsable de los bajos niveles séricos de PSA en el cáncer de próstata metastásico ${ }^{(9-11)}$.

El PSA en niveles altos ha demostrado ser un marcador confiable de cáncer de próstata metastásico, como lo demuestra el estudio de Spencer et al. (9), en el que se encontró una sensibilidad del $92 \%$ para metástasis ósea y linfática con niveles de PSA por encima de $20 \mathrm{ng} / \mathrm{mL}$. Asimismo, se recomienda no realizar tomografía ni gammagrafía ósea en pacientes con PSA $<20 \mathrm{ng} / \mathrm{mL}$ o Score de Gleason $<6^{(9,12)}$. Sin embargo, se ha observado un grupo de pacientes con cáncer de próstata metastásico (menos del 1\%) con niveles de PSA menores a $10 \mathrm{ng} / \mathrm{mL}$. Estos son llamados PSA negativos $(6,12,13)$.

Existe escasa información sobre este tipo de pacientes. Birtle et al. ${ }^{(6)}$, revisaron una serie británica de 33 pacientes con carcinoma de próstata metastásico y niveles de PSA $<10 \mathrm{ng} / \mathrm{mL}$. El promedio de edad de los pacientes fue 67 años (rango: $48-81$ años); $51 \%$ tuvo síntomas urinarios o dolor pélvico; $21 \%$ dolor óseo; $18 \%$ metástasis ganglionar abdominal; $9 \%$ hipercalcemia; $6 \%$ infiltración de médula ósea, o caquexia. El valor promedio de PSA fue de 3,5 ng/mL (rango 0,1 - 9,8 ng/mL). En forma similar, a los pacientes con PSA elevado, el $81 \%$ presentó metástasis ósea con compromiso del esqueleto axial $(48 \%)$, pelvis $(27 \%)$ y fémur $(24 \%)$. Asimismo, hubo metástasis visceral en dos pacientes (pulmones e hígado) y el score de Gleason fue 8-10 en 76\% y 6-7 en el $24 \%$ de los pacientes ${ }^{(6)}$.

En el caso presentado, el paciente tuvo un PSA de $4 \mathrm{ng} / \mathrm{mL}$ al momento del diagnóstico. Presentó características similares en relación a la edad, con dolor óseo por metástasis a hueso sacro y pérdida ponderal de $15 \mathrm{~kg}$. Asimismo, el Score de Gleason de 8, lo cual nos indica un tumor indiferenciado de crecimiento rápido y con desarrollo de neovascularización concordante con el estado avanzado de la enfermedad.

Los casos de cáncer de próstata metastásico con PSA menores de $10 \mathrm{ng} / \mathrm{mL}$ tienden a ser de mal pronóstico debido a su agresividad y pobre respuesta a la ablación hormonal. El manejo terapéutico se dificulta debido a la falta de un marcador sérico confiable ${ }^{\left({ }^{9}\right.}$. En la serie de casos descrita anteriormente, inicialmente todos los pacientes recibieron manipulación hormonal con una mejoría subjetiva del $73 \%$. El beneficio fue relacionado con la reducción del dolor y el uso de dosis menores de analgésicos o mejoría del estado general. Se observó una respuesta adecuada hasta los 14 meses de seguimiento y los valores de PSA disminuyeron en seis pacientes pero su correlación con la mejoría clínica solo se observó en dos de ellos. El tiempo promedio de sobrevida fue de 12 meses (rango: $1-36$ meses) ${ }^{(6)}$.

El caso reportado presentó una evolución similar a lo descrito en la literatura, con respuesta subjetiva al bloqueo hormonal por mejoría de dolor y del peso. Sin embargo, la enfermedad prosiguió y se decidió aplicar radioterapia. 
Se concluye que un nivel bajo de PSA (menores de $10 \mathrm{ng} / \mathrm{mL}$ ) no descarta la posibilidad de cáncer de próstata metastásico. Recomendamos que en pacientes con síndrome consuntivo y dolor sacro, se realicen estudios de imágenes para descartar cáncer de próstata que puede presentarse con valores bajos de PSA.
Contribuciones de autoría: ambos autores participaron en todo el proceso de elaboración del reporte de caso.

Fuentes de financiamiento: autofinanciado.

Conflictos de interés: los autores declaran no tener conflictos de interés en la publicación de este artículo.

\section{REFERENCIAS BIBLIOGRÁFICAS}

1. Jemal A, Siegel R, Ward E, Hao Y, Xu J, Murray T, et al. Cancer statistics, 2008. CA Cancer J Clin. 2008; 58(2):71-96.

2. European Association of Urology. EAU Clinical Guidelines 2012 [Internet]. The Netherlands: EAU [citado el 22 de abril de 2012]. Disponible en: http://www. uroweb.org/guidelines/eau-clinicalguidelines/

3. Gjertson CK, Albertsen PC. Use and assessment of PSA in prostate cancer. Med Clin N Am. 2011;95(1):191-200.

4. Andriole GL, Crawford ED, Grubb RL 3rd, Buys SS, Chia D, Church TR, et al. Mortality results from a randomized prostate-cancer screening trial. N Engl J Med. 2009; 360(13):1310-9. Erratum in: N Engl J Med. 2009; 360(17):1797.

5. Schröder FH, Hugosson J, Roobol MJ, Tammela TL, Ciatto S, Nelen $\mathrm{V}$, et al. Screening and prostatecancer mortality in a randomized European study. N Engl J Med. 2009;360(13):1320-8.

6. Birtle AJ, Freeman A, Masters JR, Payne HA, Harland SJ; BAUS Section of Oncology Cancer Registry. Clinical features of patients who present with metastatic prostate carcinoma and serum prostate-specific antigen (PSA) levels < $10 \mathrm{ng} / \mathrm{mL}$ : the "PSA negative" patients. Cancer. 2003;98(11):2362-7.

7. Catalona WJ, Richie JP, Ahmann FR, Hudson MA, Scardino PT, Flanigan RC, et al. Comparison of digital rectal examination and serum prostate specific antigen in the early detection of prostate cancer: results of a multicenter clinical trial of 6,630 men. J Urol. 1994;151(5):1283-90.

8. Thompson IM, Pauler DK, Goodman PJ, Tangen CM, Lucia MS, Parnes $\mathrm{HL}$, et al. Prevalence of prostate cancer among men with a prostate-specific antigen level $<$ or $=4.0 \mathrm{ng}$ per milliliter. N Engl J Med. 2004;350(22):2239-46.

9. Spencer JA, Chng WJ, Hudson E, Boon AP, Whelan P. Prostate specific antigen level and Gleason score in predicting the stage of newly diagnosed prostate cancer. Br J Radiol. 1998;71(851):1130-5.
10. Lawrentschuk N, Webb DR, Mitchell CA. Metastatic prostate cancer to lung with normal prostate specific antigen levels. Hosp Med. 2004;65(2):116-7.

11. Nishio R, Furuya Y, Nagakawa O, Fuse H. Metastatic prostate cancer with normal level of serum prostatespecific antigen. Int Urol Nephrol. 2003;35(2):189-92.

12. Birtle AJ, Freeman A, Masters JR, Payne HA, Harland SJ; BAUS Section of Oncology Cancer Registry. Tumour markes for managing men who present with metastatic prostate cancer and serum prostate-specific antigen levels of $<10$ ng/ml. BJU Int. 2005;96(3):303-7.

13. Balk SP, Ko YJ, Bubley GJ. Biology of prostate-specific antigen. J Clin Oncol. 2003;2182): 383-91.

Correspondencia: Silvia Diaz Velarde Dirección: Pasaje Mario Urteaga 139, etapa II de las Torres de San Borja, Lima 41, Perú. Teléfono: (51) 999491471

Correo electrónico: silviajanetdiaz@hotmail. com
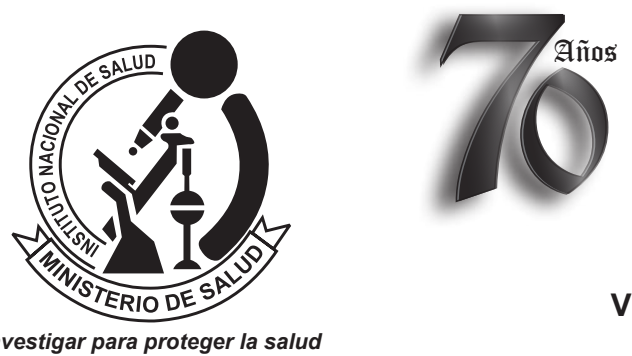

REVISTA PERUANA DE MEDICINA EXPERIMENTAL Y SALUD PÚBLICA CUMPLIENDO SUS METAS Y PROYECTÁNDOSE AL FUTURO

\section{Visite los contenidos de la revista en: www.ins.gob.pe/rpmesp}

